\title{
Developing Consensus on Appropriate Standards of Hospital Disaster Care: Ensuring That the Needs of Children Are Addressed
}

\author{
David Markenson, MD, FAAP, EMT-P
}

$\mathrm{I}$ $\mathrm{n}$ this issue of Disaster Medicine and Public Health Preparedness, Kanter et al present a method for "developing consensus on hospital disaster interventions that should be regarded as essential, quantitatively balancing needs and resources." 1 The authors begin by discussing the importance of establishing consensus-based guidelines for altered standards of care and the interventions that should be provided during times of a disaster when patients and their needs will exceed available resources. Their points regarding the need to prospectively develop an approach with which interventions can be provided and the maximal amount of clinical effectiveness that staff can be expected to provide are important. In addition, they are correct that alterations in standard patient care in disaster response, and in some situations such as pandemic influenza sustaining these alterations for a prolonged period of time, would represent a virtually unprecedented shift for clinicians, public officials, patients, and families. The method presented not only provides a sound method for emergency planning but also will allow government entities, regulatory and credentialing organizations, and professional organizations to prospectively define appropriate disaster standards of care. In so doing, they will afford providers legal protection and clear direction for the decisions they make during a disaster.

Although it will be difficult for providers to alter care for any patient, it may be difficult if not impossible for them to do this for children during a disaster without clear prospectively developed recommendations and methodologies. This is due to multiple reasons which include the societal expectation for care of children, the emotional burden of potentially limiting or withholding care from a child, and the unique barriers faced when trying to provide care for children. Therefore, as a society we need to develop clear recommendations for the hospital care of children that address both intensive care unit (ICU) and non-ICU level of care and determine which essential interventions could be provided with the available resources that at a minimum include space, equipment, pharmaceuticals, and providers.
The authors, after describing their methodology for this consensus approach, describe its application to recommendations for children in disasters. Specifically, they discuss the usage of only selected interventions that can be accomplished with available resources during a disaster for children both in an ICU and a non-ICU setting. I commend the authors for applying this new methodology to the needs of children. In my group's previous work on the subject of consensus-based emergency preparedness guidelines for children, we also recognized the need for clear guidelines for pediatric preparedness, the importance of giving clinicians and emergency management providers guidance as to the needs of children, and allowing planning efforts to incorporate these targeted needs in all aspects of emergency planning. ${ }^{2,3}$

It should be recognized that, although limited in scope and only an initial effort, the recommendations developed by Kanter et al do present a good foundation for the development of future guidelines for children, and can serve in their current form as the foundation for planning for adult populations in times of disaster. But the work done does not fully address the unique aspects of children and thus would limit the application of the recommendations for use in the planning of standards of hospital care for children in disasters.

As multiple previous authors have documented ${ }^{4-6}$ there are unique pediatric considerations that must be accounted for in disaster planning for children. The categories of issues which must be addressed at a minimum include the following:

Unique vulnerabilities of children to disasters, terrorism and public health emergencies

Limited pediatric specific resources

Equipment

Pharmaceuticals

Providers

Federal resources (Disaster Medical Assistance Teams, Strategic National Stockpile)

Adult care providers caring for children 
Pediatric care in adult units with adult-specific equipment Pediatric surge

Modification of procedures for children (eg, decontamination)

A full elaboration and description of each of the categories listed above would be beyond the scope of this editorial, but a few examples that follow demonstrate the need for incorporation of pediatric considerations when developing hospital and critical care recommendations for children in times of disasters. When one considers pediatric surge one must plan for different forms of surge. One type of surge would have children affected along with the rest of the population in which the surge of pediatric patients would be equal to their proportion in the community affected but may be slightly higher based on unique vulnerabilities to certain events. The other form of pediatric surge would be one in which either the majority of victims or all of the victims are children due to an event that either targets children or occurs at location where children congregate, such as a school.

The authors present a scenario in which the entire surge of 500 patients per million population (which is the standard number used for most community surge planning) would be children. Although an entirely pediatric event is one any community may have to deal with, it would require several other modifications not addressed in this article. The planning assumption communities have been asked to make is the ability to handle a surge of 500 patients per million. ${ }^{7}$ Emergency planners assume this to be a mixture of pediatric and adult patients usually in a ratio consistent with the ratio in their community. If this basic planning assumption were to change to all pediatric patients, then this would require alteration in hospital disaster plans that would include at a minimum adult providers to care for pediatric patients in adult units with adult equipment. In such cases, measures of staff workload such as the Therapeutic Intervention Scoring System (TISS) would not be improved due to disaster-imposed approaches, but in fact would be reduced. This would be due to multiple barriers including adult providers' lack of familiarity with pediatric patients, the care and procedures performed on children, and challenges with child-specific issues ranging from medication dosing to meeting the nutritional needs of children from birth through adolescence. As such, the interventions advocated, although minimal, may not be possible because the true TISS would be higher in the setting of a uniquely pediatric event, thus reducing the actual interventions that a planner could recommend providing.

As has been described in previous articles, caring for children can be technically challenging due to multiple reasons including their smaller size and unique anatomy and physiology. ${ }^{8,9}$ In addition, interventions for children can also be time consuming due to children's understanding of procedures to be performed and their compliance with these interventions. The implication of these pediatric specific issues is that the same interventions performed on children in general will require more staff resources and thus pediatric- specific TISS values or development of a pediatric-specific staff resource tool.

Finally, as has been described previously, ${ }^{4,6}$ children have unique vulnerabilities to disasters, terrorism, and public health emergencies. It has been further described that these unique vulnerabilities would not only lead to increased numbers of children affected but also each child could be more severely affected when compared with adults. If in fact children would be sicker and/or more severely injured than adults, then the care each child would need would be greater than that for an adult on average. This would require alteration of standard disaster planning assumptions of the factor to which existing patient staff ratios could be expanded. As Kanter et al describe, this is assumed to be a factor of 4 adults but may need to be reduced to only 2- or 3-fold for children.

Clearly, as Kanter et al discuss, it is imperative to reach consensus on essential interventions. The methodology that they present to develop evidence- and resource-based guidelines for disaster preparedness is one which needs to be applied broadly to further enhance disaster planning by focusing efforts on the intersection between available resources, modifications and alterations in standards of care during disaster times, and the needs of the affected population.

In addition, the needs of children must be accounted for in hospital disaster care planning by use of this excellent staff resource methodology in combination with a broader expertise group and taking into account all of the previously described unique pediatric considerations. This would require validation and modification of the TISS approach to children in disasters, accounting for everything from alterations due to difficulties in performing procedures on children through changes in efficiency with adult providers caring for pediatric patients in adult units with adult-specific equipment. If some of these unique pediatric considerations had been included in the assumptions of the application of their recommendation methodology, I am certain their excellent methodology would have concluded that there are multiple differences in ICU and overall hospital essential interventions in times of disasters for children when compared with adults.

By using the approach to resource utilization and rationing described in this article, combined with taking into account the unique aspects of pediatric care, we can improve our planning and responses. This can be accomplished by understanding the needs of the population being served, learning how to focus on both pediatric needs and the expectations of the community with regard to the care of children, adopting what has been learned in prior events in the United States and abroad, and developing prospective recommendations regarding essential interventions that can be performed in a disaster. This will help the provider faced with ill and injured children triage scarce resources, which is difficult to do with any patient population but emotionally and culturally more difficult when faced with limiting care and altering the standards of care for a child. In so doing, we can better develop 
an appropriate model for response to and recovery from future disasters that may occur and ensure that the resources we have provide the most benefit to as many children as possible.

\section{About the Author}

Dr Markenson is Director, Center for Disaster Medicine, New York Medical College.

Address correspondence and reprint requests to Dr David Markenson, Center for Disaster Medicine, New York Medical College, Suite 100, Valhalla, NY 10595 (e-mail: david_markenson@nymc.edu).

Received for publication October 1, 2008; accepted October 6, 2008.

\section{Author's Disclosure}

The author reports no conflicts of interest.

ISSN: 1935-7893 (C) 2009 by the American Medical Association and Lippincott Williams \& Wilkins.

DOI: 10.1097/DMP.0b013e318190a2a7

\section{REFERENCES}

1. Kanter RK, Andrake JS, Boeing NM, et al. Developing consensus on appropriate standards of disaster care for children. Disaster Med Public Health Preparedness. 2009;3:27-32.
2. Markenson D, Reilly M, Amler R, et al. Emergency Preparedness Guidelines for Children: Revised February 2008. New York: Center for Disaster Medicine, New York Medical College School of Public Health; 2008.

3. Markenson D, DiMaggio C, Redlener I. Pediatric terrorism preparedness national guidelines and recommendations: findings of an evidence-based consensus process. Biosecur Bioterror. 2004;2:301-319.

4. Foltin GL, Schonfeld DJ, Shannon MW, et al. Pediatric Terrorism and Disaster Preparedness: A Resource for Pediatricians. AHRQ publication 06(07)-0056. Rockville, MD: Agency for Healthcare Research and Quality; 2006.

5. Pediatric Disaster Toolkit: Hospital Guidelines for Pediatrics in Disasters. New York City, 2006. New York City Department of Health and Mental Hygiene Web site. www.nyc.gov/html/doh/html/bhpp/bhpp-focus-ped-toolkit.shtml. Accessed April 13, 2007.

6. Markenson D, Reynolds S. The pediatrician and disaster preparedness. Pediatrics. 2006;117:e340-e362.

7. Office of the Assistant Secretary for Preparedness and Response, FY 2009 Online Performance Appendix. http://wwww.hhs.gov/budget/09budget/asprfy09opa. pdf. Accessed January 17, 2009.

8. Markenson D. The treatment of children exposed to pathogens linked to bioterrorism. Infect Dis Clin North Am. 2005;19:731-745.

9. American Academy of Pediatrics Committee on Environmental Health and Committee on Infectious Diseases . Chemical-biological terrorism and its impact on children. Pediatrics. 2006;118:1267-1278. 